\title{
Pericardial-Esophageal Fistula: A Rare but Increasing Complication of Cardiac Ablation
}

\author{
Erik A. Sylvin ${ }^{1} \quad$ Arminder S. Jassar ${ }^{2} \quad$ John C. Kucharczuk ${ }^{3}$ Prashanth Vallabhajosyula ${ }^{4}$
}

\author{
${ }^{1}$ Division of Cardiothoracic Surgery, JFK Medical Center, University of \\ Miami Miller School of Medicine, Atlantis, Florida, United States \\ 2 Division of Cardiac Surgery, Department of Surgery, Massachusetts \\ General Hospital, Boston, Massachusetts, United States \\ ${ }^{3}$ Division of Thoracic Surgery, Hospital of the University of \\ Pennsylvania, Philadelphia, Pennsylvania, United States \\ ${ }^{4}$ Section of Cardiac Surgery, Yale University School of Medicine, \\ New Haven, Connecticut, United States
}

Thorac Cardiovasc Surg Rep 2022;11:e27-e29.

\author{
Address for correspondence Erik Sylvin, MD, MS, Division of \\ Cardiothoracic Surgery, JFK Medical Center, University of Miami Miller \\ School of Medicine, Atlantis 33462, FL, United States \\ (e-mail: erik.sylvin@hcahealthcare.com).
}

\author{
Abstract \\ Keywords \\ - surgical ablation \\ - esophageal \\ - pericardium \\ - cardiopulmonary \\ bypass \\ - CPB
}

Pericardial-esophageal fistula and/or atrial-esophageal fistula after cardiac ablation is nearly universally fatal if not detected and treated expeditiously. This condition should be assumed and ruled out in anyone with a recent history of cardiac ablation presenting with signs of sepsis, pneumomediastinum, pneumopericardium, or chest pain. Computed tomography scan of the chest is a rapid and a sensitive diagnostic modality. Tenets of treatment and repair consist of preventing an air embolism, repairing the esophageal perforation and atrial defect, and interposing autologous tissue between the esophagus and heart.

\section{Case History}

The patient was a 35-year-old woman with atrial fibrillation (AF) for 6 years. She underwent several electrical cardioversion procedures in the past. She has reverted to persistent symptomatic AF. She was electrically cardioverted and underwent successful transcatheter radiofrequency ablation. Two weeks after discharge, she presented to another facility with low-grade fever, general malaise, and substernal chest pain. Computed tomography (CT) scan demonstrated intrapericardial air and fluid ( - Fig. 1). Given her history, we suspected a pericardial-esophageal fistula (PEF) or a possible atrial-esophageal fistula (AEF) and emergently transferred her to our center. She was expeditiously transferred to the operating room. She was maintained in Trendelenburg position during this entire process to avoid air embolus in the event of an AEF. Both cardiac and general thoracic teams operated on this patient in concert.

received

April 1, 2021

accepted

June 16, 2021
A midline sternotomy and then pericardiotomy was performed. Purulent material and food particles were noted. Ascending aortic and bicaval cannulation were performed and cardiopulmonary bypass (CPB) was initiated. The ascending aorta was cross-clamped and antegrade cardioplegia was administered to obtain cardiac arrest ( - Fig. 2). The heart was lifted up to expose the posterior pericardium and left atrium, and a fistula from the esophagus to the pericardium was identified (-Fig. 3). The left atrium was carefully separated from the esophageal defect and was noted to be indurated and fibrotic; however, the left atrium was not perforated and there were no areas of bleeding. Esophagoscopy was performed to carefully evaluate the esophageal defect ( $\mathbf{F i g . ~ 4 ) . ~ T h i s ~ w a s ~}$ debrided to healthy tissue, separating the esophageal mucosa from the muscle layers. This was closed primarily in two layers with several interrupted 3-0 Vicryl sutures and buttressed with pericardium. The esophagus was insufflated endoscopically

\footnotetext{
(C) 2022. The Author(s).

This is an open access article published by Thieme under the terms of the Creative Commons Attribution-NonDerivative-NonCommercial-License, permitting copying and reproduction so long as the original work is given appropriate credit. Contents may not be used for commercial purposes, or adapted, remixed, transformed or built upon. (https://creativecommons.org/ licenses/by-nc-nd/4.0/) Georg Thieme Verlag KG, Rüdigerstraße 14, 70469 Stuttgart, Germany
} 


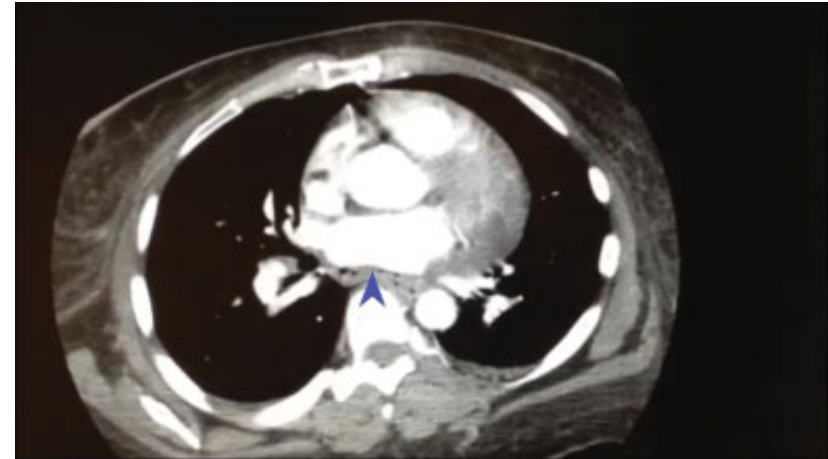

Fig. 1 A computed tomography scan with intravenous contrast was performed which demonstrated intrapericardial air adjacent to the left atrium (arrow). No other imaging studies were obtained.

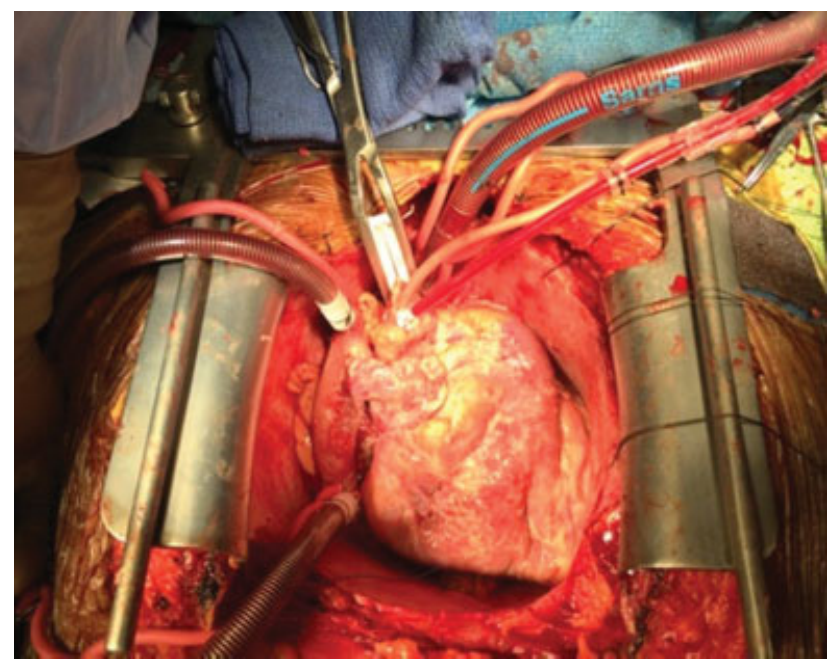

Fig. 2 The approach was via a sternotomy and laparotomy. Minimal manipulation of the heart occurred before bicaval cannulation and aortic cross-clamp to prevent air embolism.

and the repair was pneumostatic. Next, an upper midline laparotomy was performed. A long, pedicled flap of omentum based on the right gastroepiploic artery was developed (-Fig. 5). The omentum was brought into the chest through an iatrogenically created defect in the central tendon of the diaphragm. It was placed posterior to the heart to separate the left atrium from the esophagus ( $\mathbf{F i g . 6}$ ). A feeding jejunostomy tube and a nasogastric tube were inserted. The entire chest and abdominal cavities were copiously irrigated and closed in the usual fashion, with wide drainage of both cavities. A follow-up esophagram demonstrated no contrast extravasation and complete healing without a remnant fistulous tract. The patient was discharged home and made an uneventful recovery.

\section{Discussion}

As the prevalence of AF continues to increase worldwide so does the corresponding number of catheter ablative procedures. As a result, one of the most devastating complications, $\mathrm{PEF}$ and/or AEF, has also been on the rise. ${ }^{1,2} \mathrm{PEF} / \mathrm{AEF}$ is a rare but potentially fatal complication after AF ablation. It is

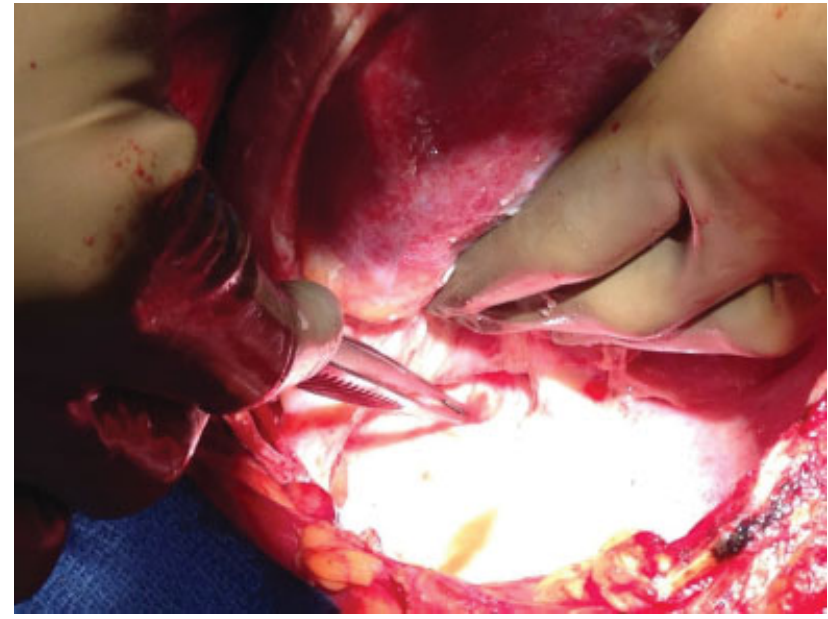

Fig. 3 With the aorta cross-clamped and the heart completely empty, the heart is lifted to reveal the communication from the esophagus to the pericardium. The left atrium appears uninvolved.

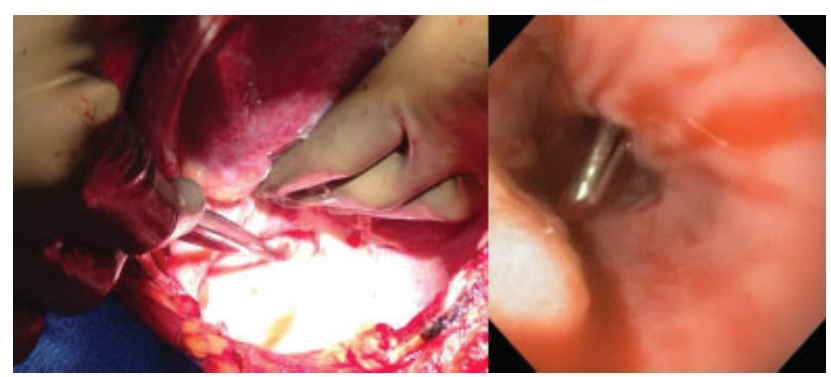

Fig. 4 Esophagogastroduodenoscopy is performed only after the aorta is cross-clamped to decrease the risk of air embolism in the event of an atrialesophageal fistula. The identification of the esophageal injury/fistula by endoscopy is facilitated by introduction of a probe from the pericardium.

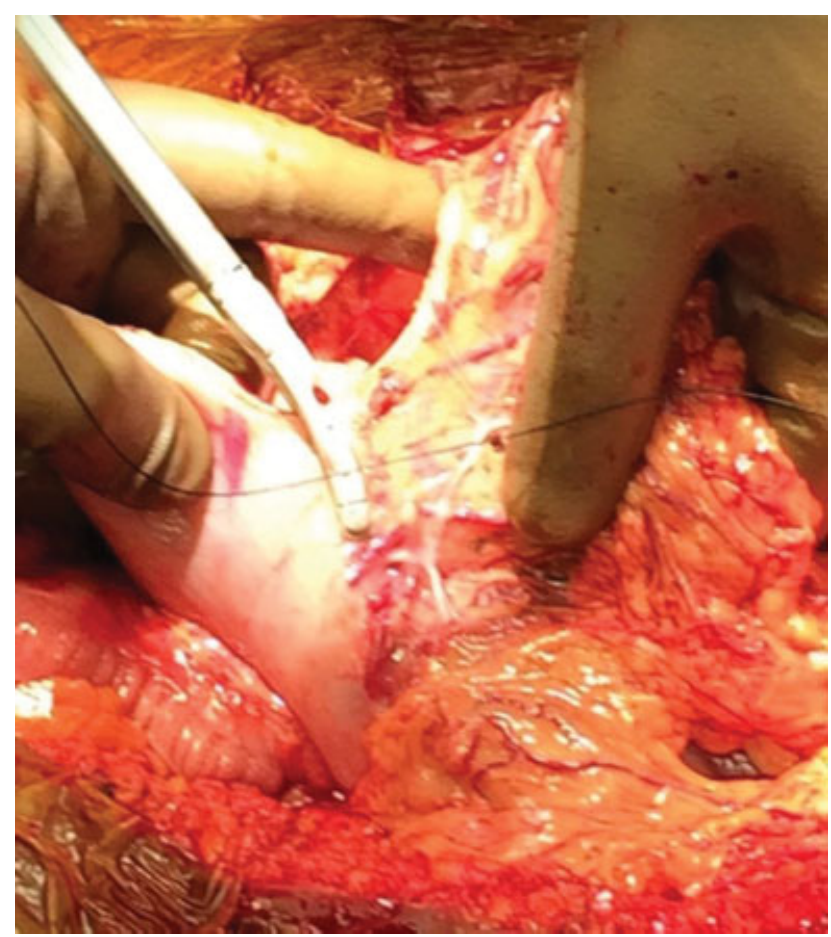

Fig. 5 Omentum was harvested (based on the right gastroepiploic artery), and brought into chest through the diaphragm. 


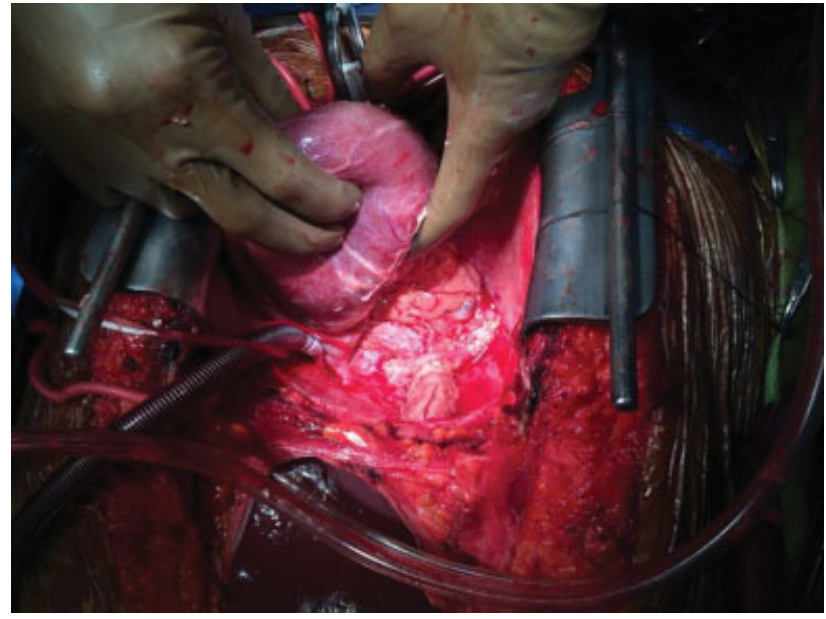

Fig. 6 The esophageal defect is primarily repaired and covered with omentum that is brought into the pericardial space through the diaphragm.

reported to occur in 0.04 to $0.25 \%$ of AF cardiac ablations. ${ }^{2,3}$ In one systematic, multicenter study involving more than 45,000 procedures, AEF was the second leading cause of death. ${ }^{4}$ No definitive risk factors have been identified predicting fistula formation. Intraoperative strategies have been proposed to help limit esophageal injury. ${ }^{2}$ These injuries have been reported with use of various energy sources such as radiofrequency ablation, cryoablation, ultrasound, and surgical ablation. ${ }^{1-3} \mathrm{~A}$ high index of suspicion must be maintained to make the diagnosis. However, the majority of patients presenting with this complication do not survive. $^{1-5}$ Potential mechanisms of injury include direct thermal injury and vagal injury and resultant increased acid reflux. Eventually, esophageal ulceration, ischemia, and necrosis develop. Patients may present with a myriad of signs and symptoms, including but not limited to chest pain, leukocytosis, fever, stroke, dysphagia, odynophagia, hematemesis, heartburn, septic or hemorrhagic shock. ${ }^{1-3,5}$ Symptom onset may occur days to months after the ablative procedure. CT scan of the chest is the test of choice to make a diagnosis of AEF or PEF. Suggestive findings include pneumopericardium, pneumomediastinum, intra-atrial air, or hemopericardium. ${ }^{1,2,5}$ In cases in which there is a connection between the left atrium and the esophagus, the patient may present with bleeding, and/or sequelae of air or food embolism. Endoscopy and esophageal insufflation and transesophageal echocardiography are avoided until after the aorta is cross-clamped to minimize risk of air embolism. It is safest to approach this condition through a sternotomy or thoracotomy, and with CPB support to deal with any fistulous connection between the esophagus and the atria that may exist. In cases where the left atrium defect cannot be closed primarily, reconstruction with bovine pericardium may be necessary.

\section{Conclusion}

Autologous tissue should be used to buttress and protect repairs. In rare cases like ours, it is imperative to collaborate with numerous subspecialists. Patients undergoing ablation should be educated about potential symptoms that may suggest a serious complication.

\section{Conflict of Interest}

None declared.

\section{References}

1 Chavez P, Messerli FH, Casso Dominguez A, et al. Atrioesophageal fistula following ablation procedures for atrial fibrillation: systematic review of case reports. Open Heart 2015;2(01):e000257

2 Pappone C, Vicedomini G, Santinelli V. Atrio-esophageal fistula after AF ablation: pathophysiology, prevention \& treatment. J Atr Fibrillation 2013;6(03):860

3 Kapur S, Barbhaiya C, Deneke T, Michaud GF. Esophageal injury and atrioesophageal fistula caused by ablation for atrial fibrillation. Circulation 2017;136(13):1247-1255

4 Cappato R, Calkins H, Chen SA, et al. Prevalence and causes of fatal outcome in catheter ablation of atrial fibrillation. J Am Coll Cardiol 2009;53(19):1798-1803

5 Zakaria A, Hipp K, Battista N, Tommolino E, Machado C. Fatal esophageal-pericardial fistula as a complication of radiofrequency catheter ablation. SAGE Open Med Case Rep 2019;7: X19841150 